\title{
2D Silicene Nanosheets for High-Performance Zinc-Ion Hybrid Capacitor Application
}

Qiang Guo ${ }^{\ddagger}, 1$, Jingjing Liu ${ }^{\ddagger}, 2$, Congcong Bai ${ }^{1}$, Nan Chen ${ }^{*, 1}$, Liangti Qu*,3

${ }^{1}$ Key Laboratory of Cluster Science, Ministry of Education of China, Key Laboratory of Photoelectronic/Electrophotonic Conversion Materials, School of Chemistry and Chemical Engineering, Beijing Institute of Technology, Beijing 100081, China

${ }^{2}$ State Key Laboratory of Infrared Physics, Shanghai Institute of Technical Physics, Chinese Academy of Sciences, 500 Yu Tian Road, Shanghai 200083, China.

${ }^{3}$ Key Laboratory of Organic Optoelectronics \& Molecular Engineering, Ministry of Education, Department of Chemistry, Tsinghua University, Beijing 100084, China

$\$$ These authors contributed equally to this work.

E-mail: gabechain@bit.edu.cn; lqu@mail.tsinghua.edu.cn.

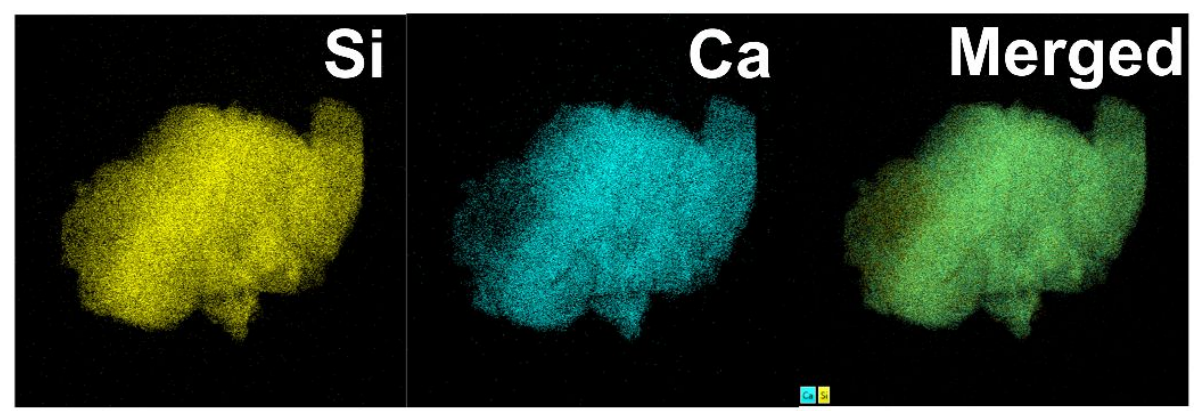

Figure S1. Elemental mapping of $\mathrm{CaSi}_{2}$. 


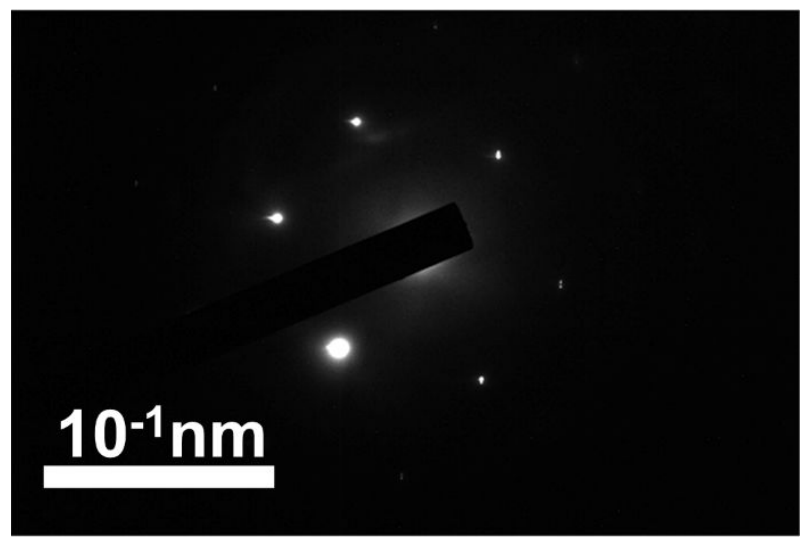

Figure S2. SAED pattern of the pristine bulk $\mathrm{CaSi}_{2}$.

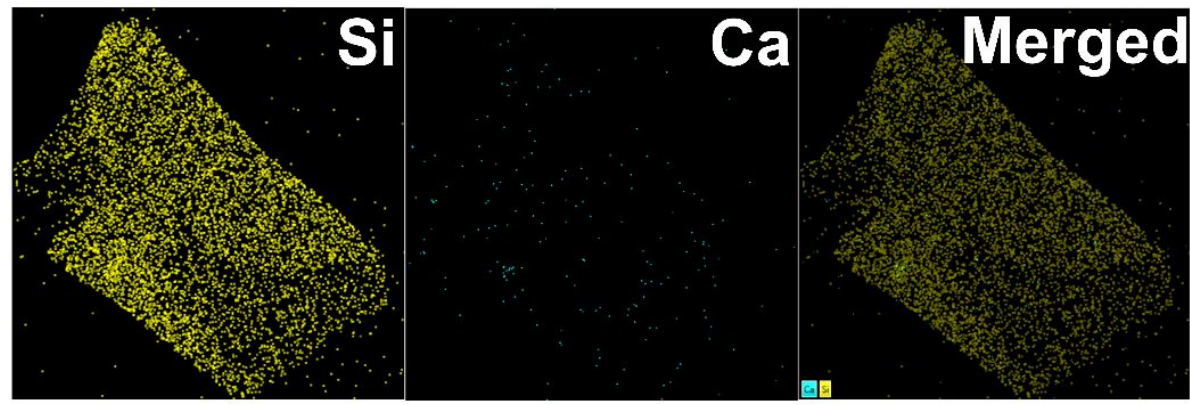

Figure S3. Elemental mapping of the as obtained silicene.

(a)

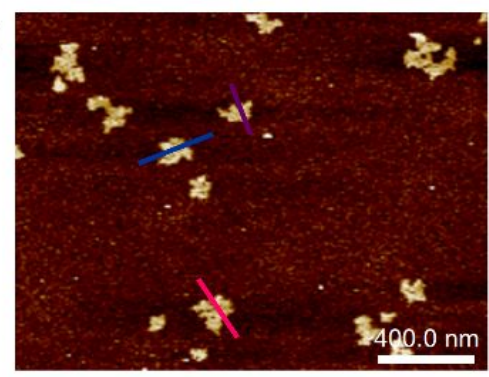

(b)

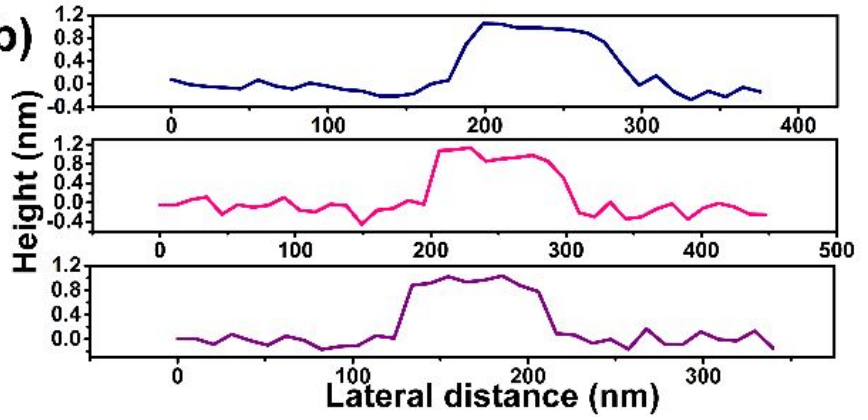

Figure S4. Thickness curves of silicene nanosheets. 


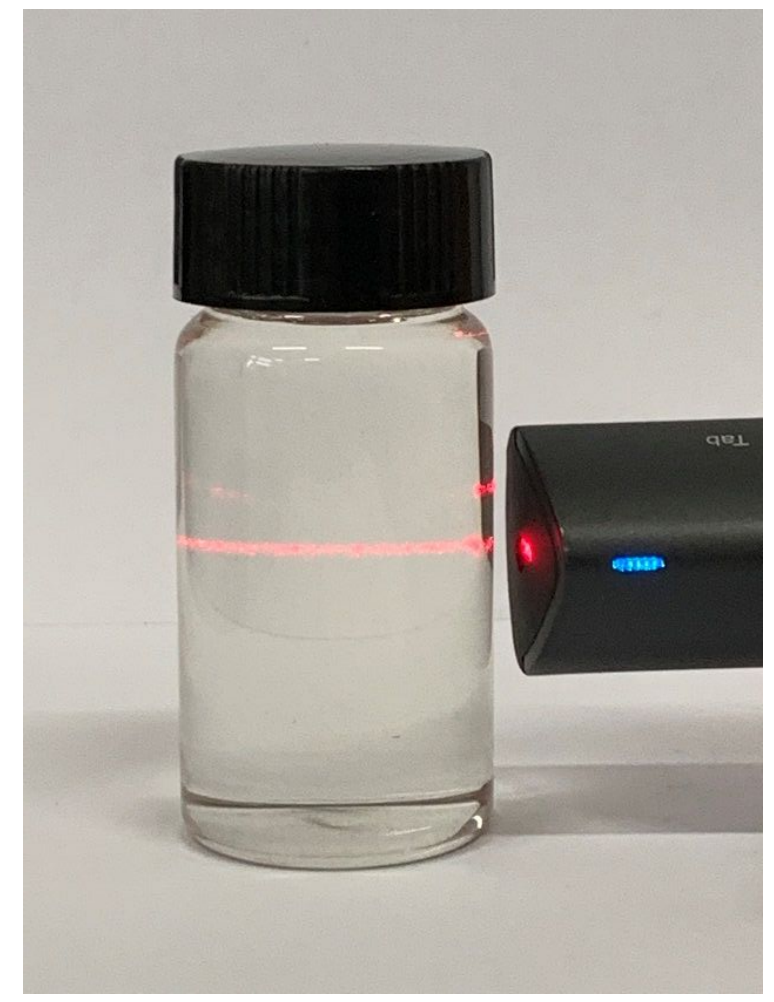

Figure S5. The stable dispersion of silicene in NMP.

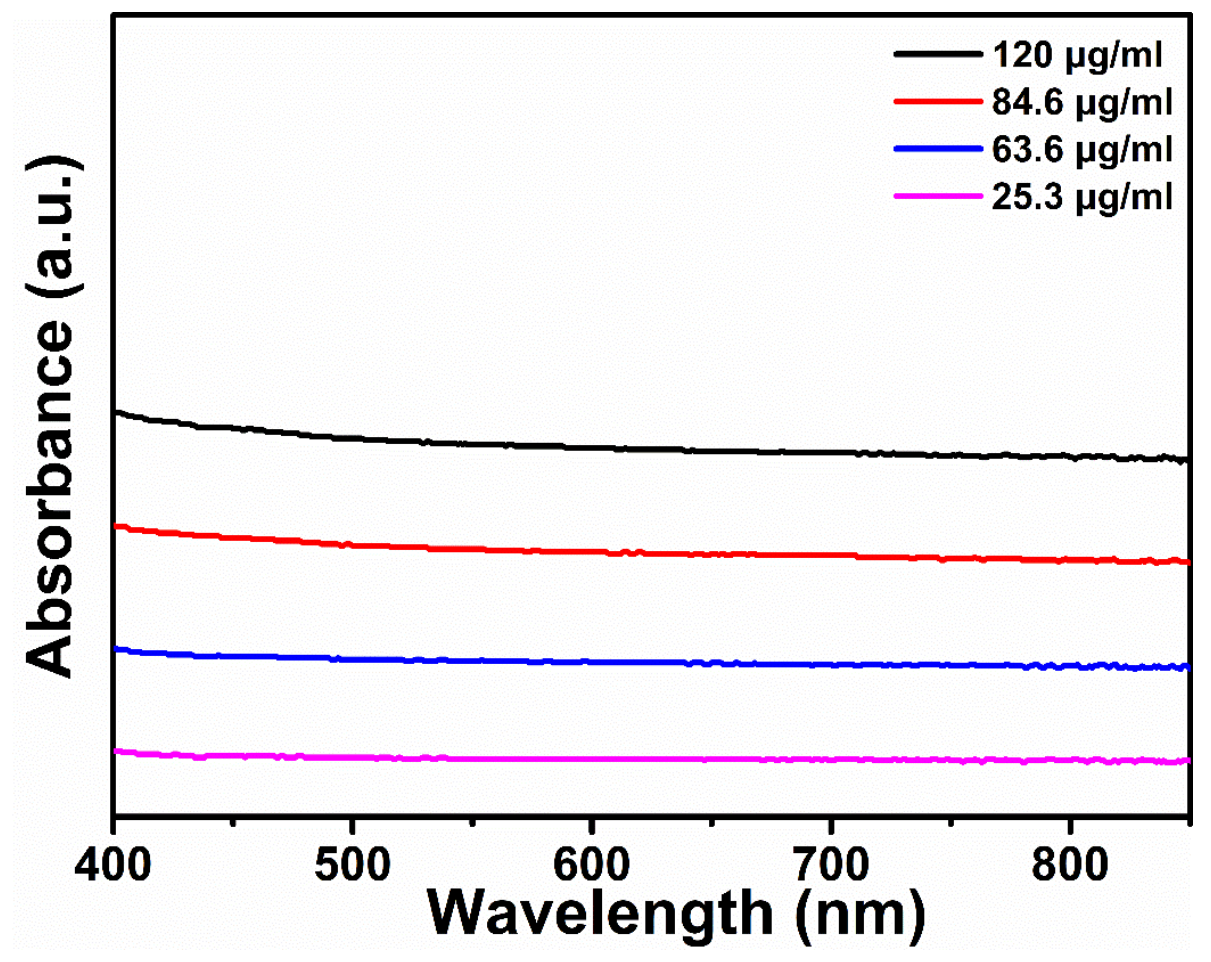

Figure S6. UV-vis-IR absorption spectra of silicene. 


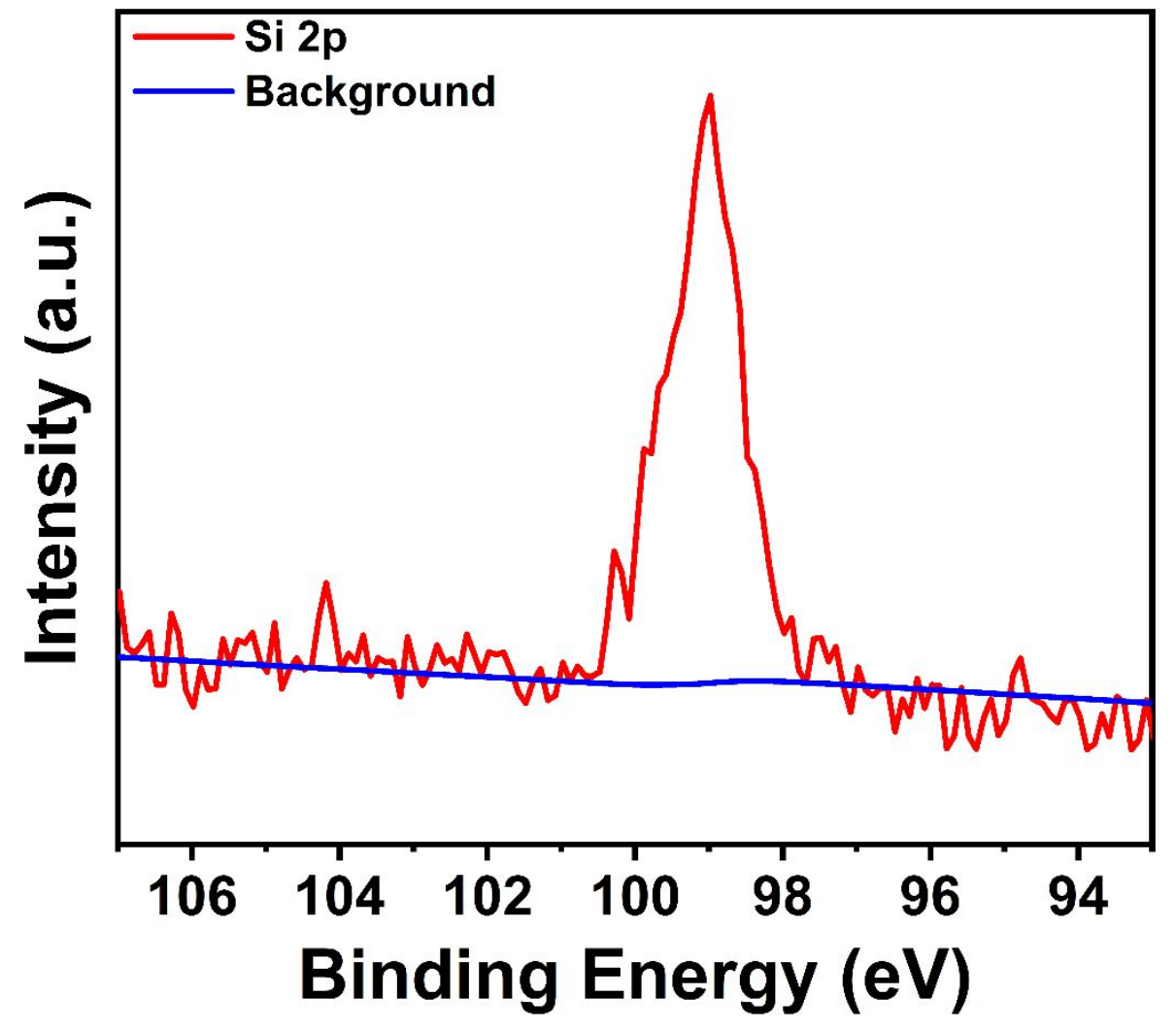

Figure S7. XPS spectrum of silicene in Si 2p region.

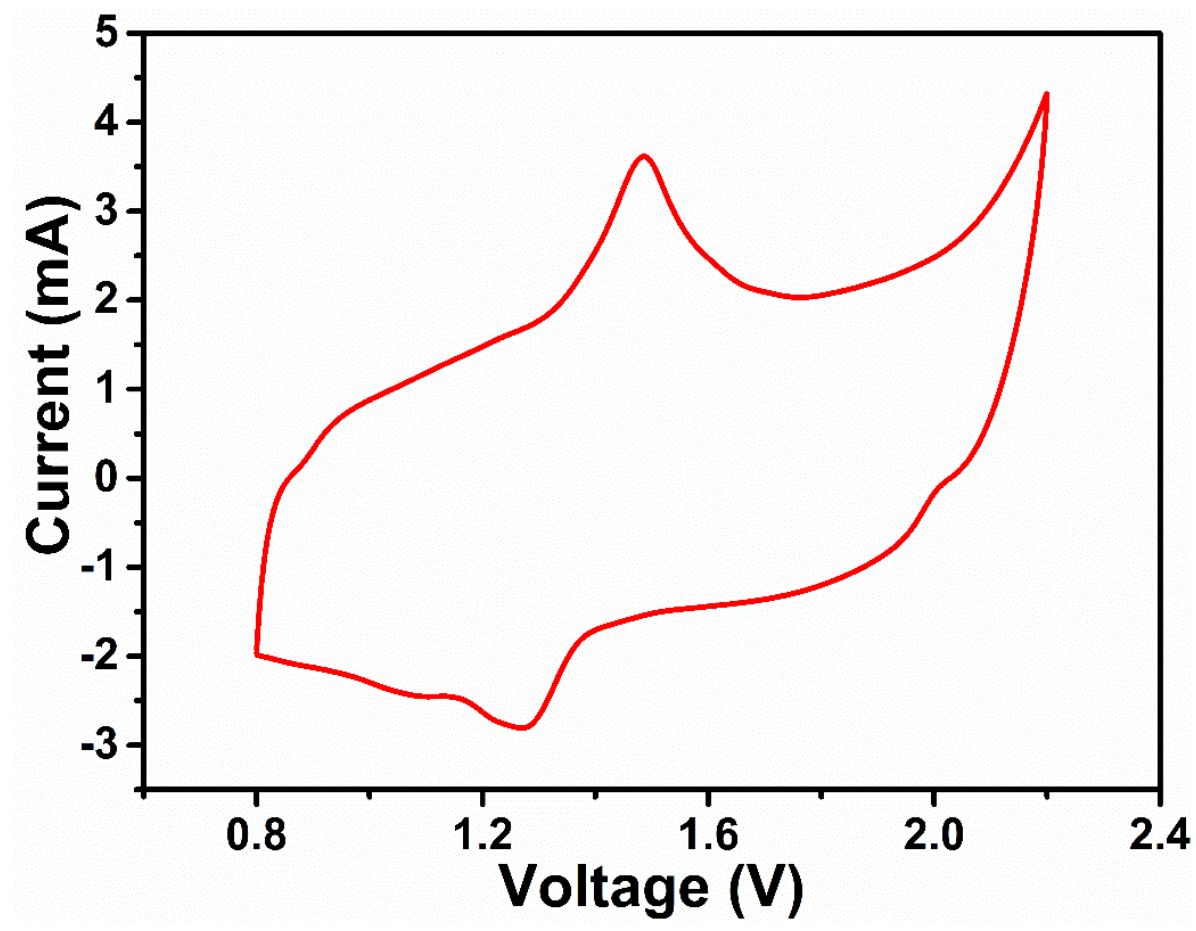

Figure S8. Cyclic voltammetric curve of the silicene ZHC at operating voltages from 0.8 to $2.2 \mathrm{~V}$ using a scan rate of $100 \mathrm{mV} \mathrm{s}^{-1}$. 


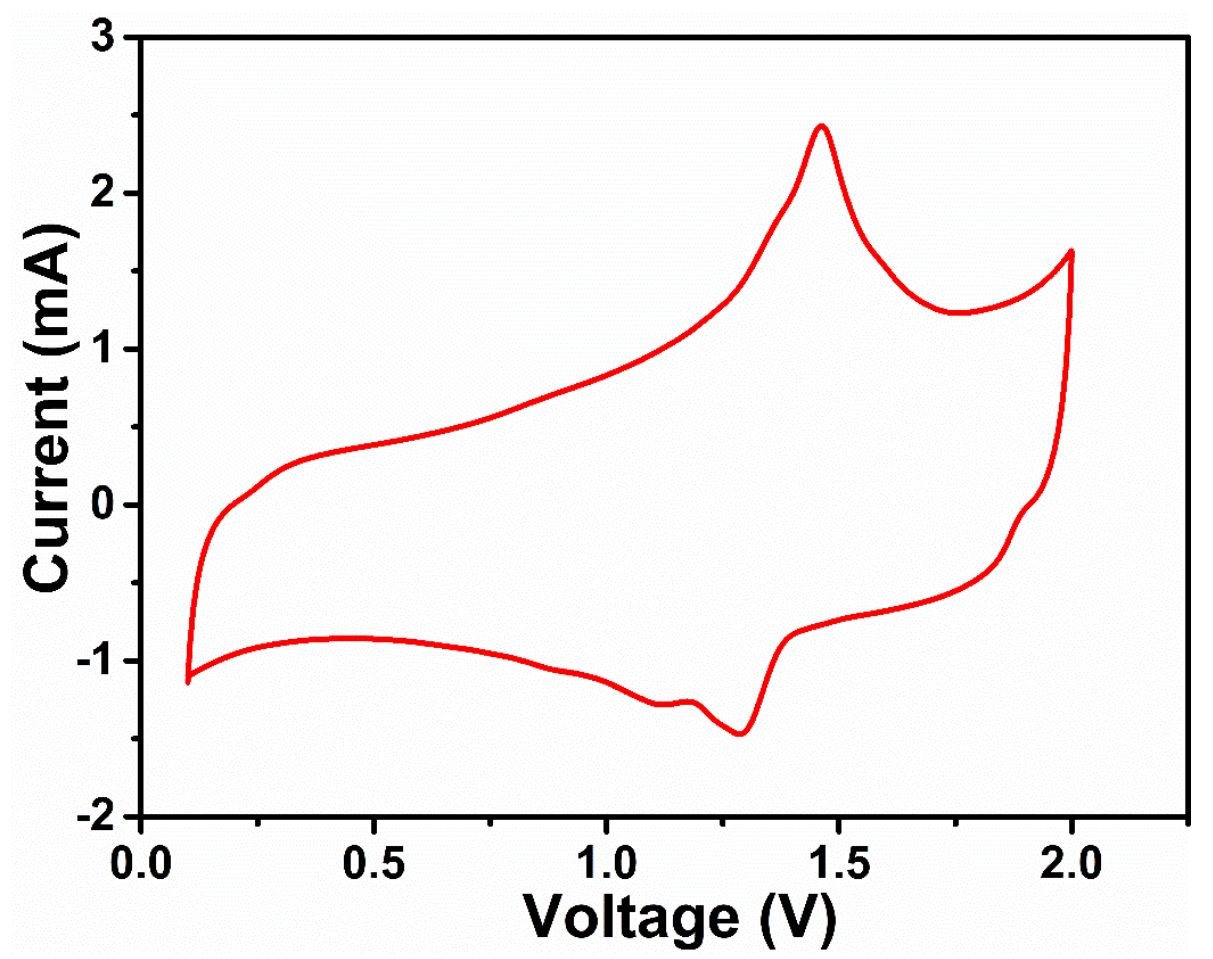

Figure S9. Cyclic voltammetric curve of the silicene ZHC at operating voltages from 0.1 to $2.0 \mathrm{~V}$ using a scan rate of $100 \mathrm{mV} \mathrm{s}^{-1}$.

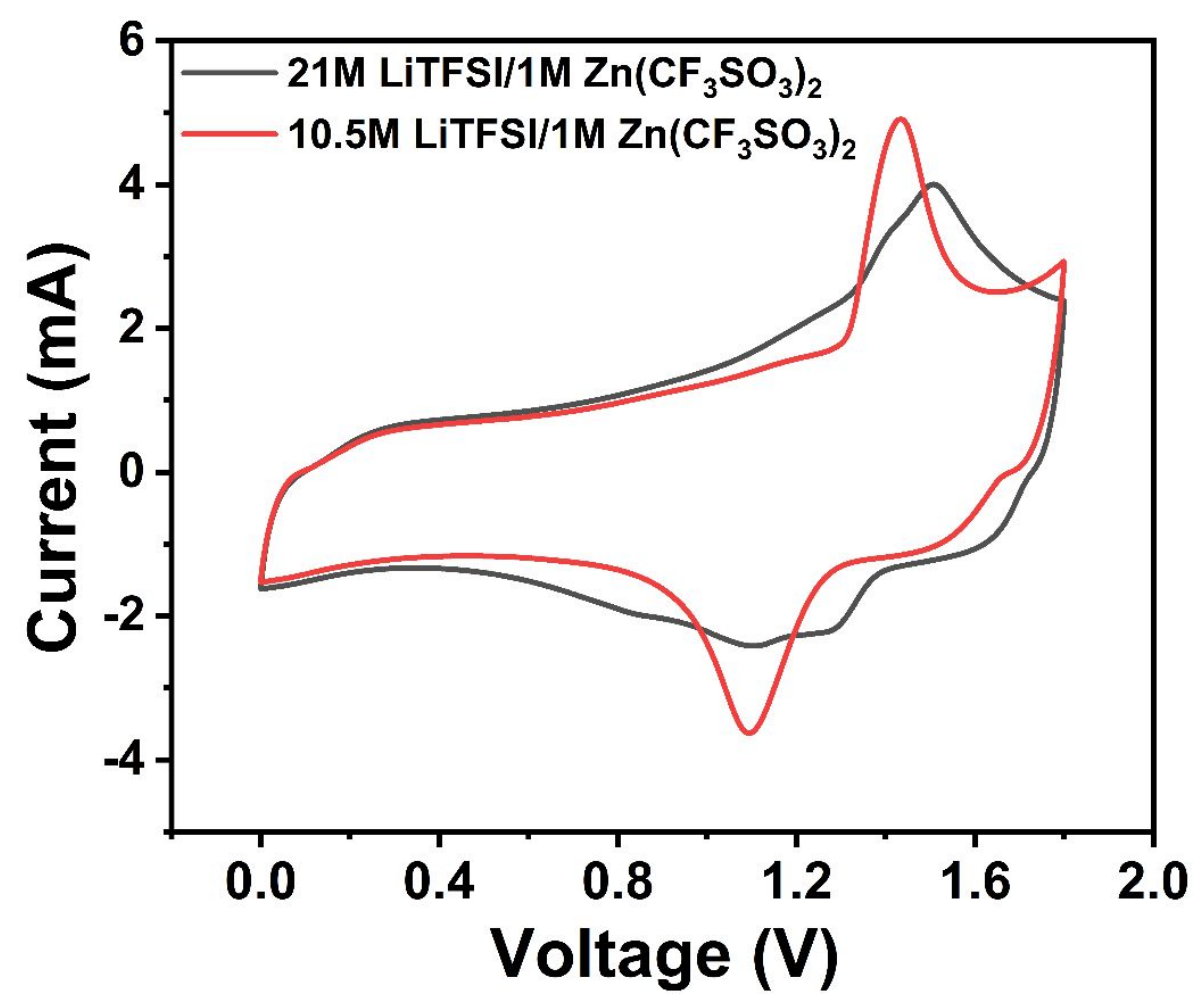

Figure S10. Cyclic voltammetric curve of the silicene ZHC using $10.5 \mathrm{M}$ LiTFSI/1 $\mathrm{M} \mathrm{Zn}\left(\mathrm{CF}_{3} \mathrm{SO}_{3}\right)_{2}$ electrolyte at operating voltages from 0.1 to $1.8 \mathrm{~V}$. 


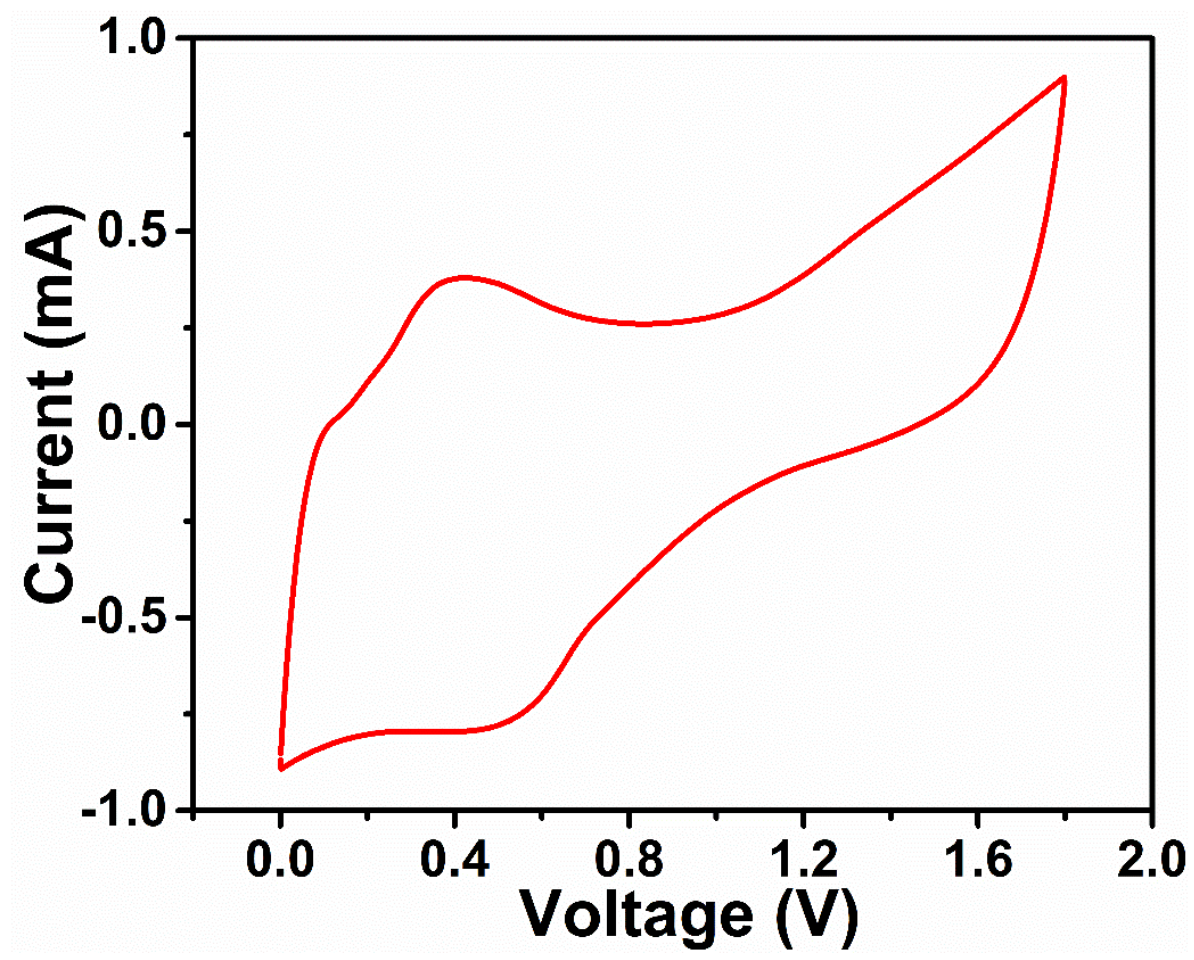

Figure S11. The CV curve of the silicene ZHC using pure $21 \mathrm{M} \mathrm{LiTFSI}$ electrolyte at a scan rate of $100 \mathrm{mV} / \mathrm{s}$.

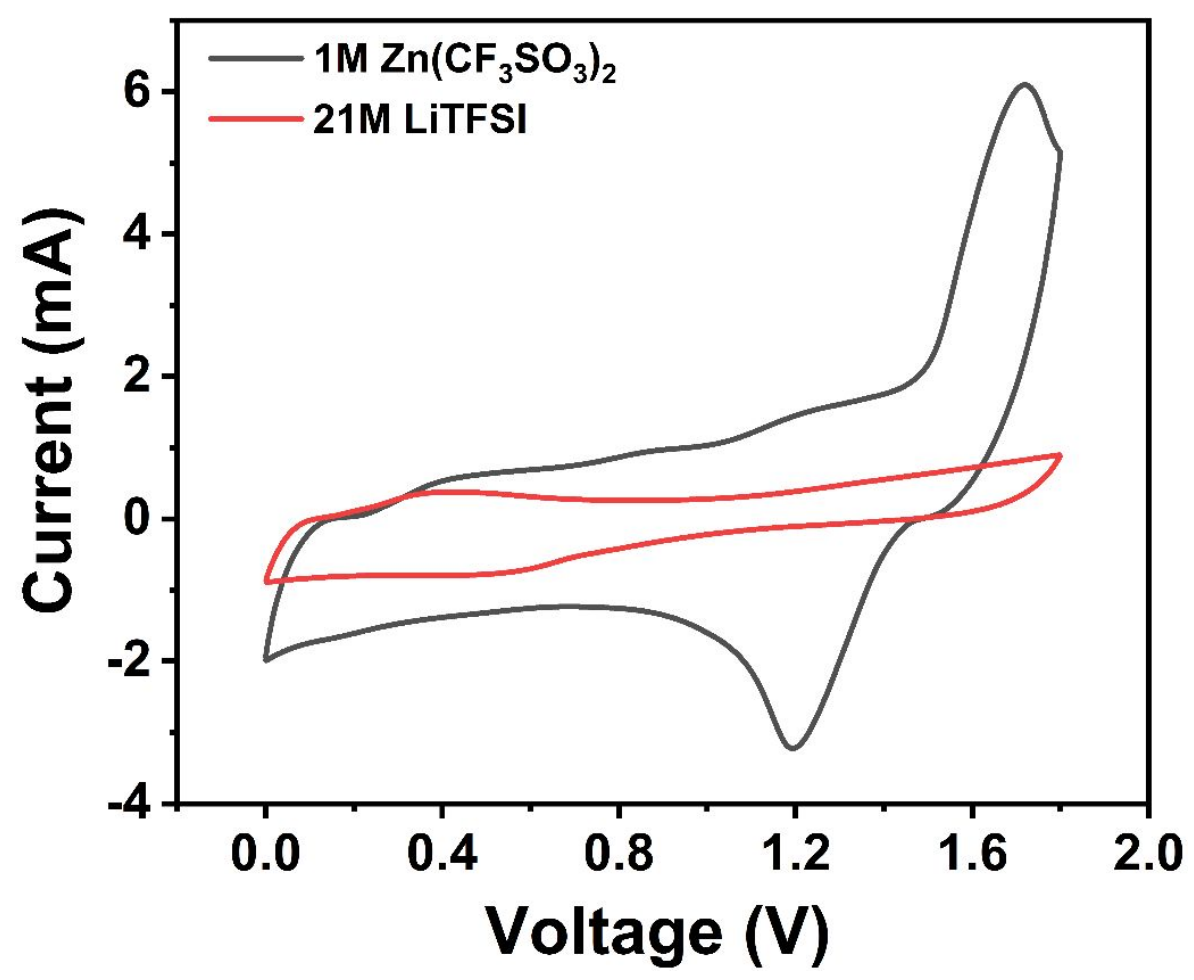

Figure S12. The CV curves of the silicene ZHCs using pure $21 \mathrm{M}$ LiTFSI and $1 \mathrm{M} \mathrm{Zn}\left(\mathrm{CF}_{3} \mathrm{SO}_{3}\right)_{2}$ electrolytes at a scan rate of $100 \mathrm{mV} / \mathrm{s}$. 




Figure S13. The effect of scan rate on anodic and cathodic peak current.

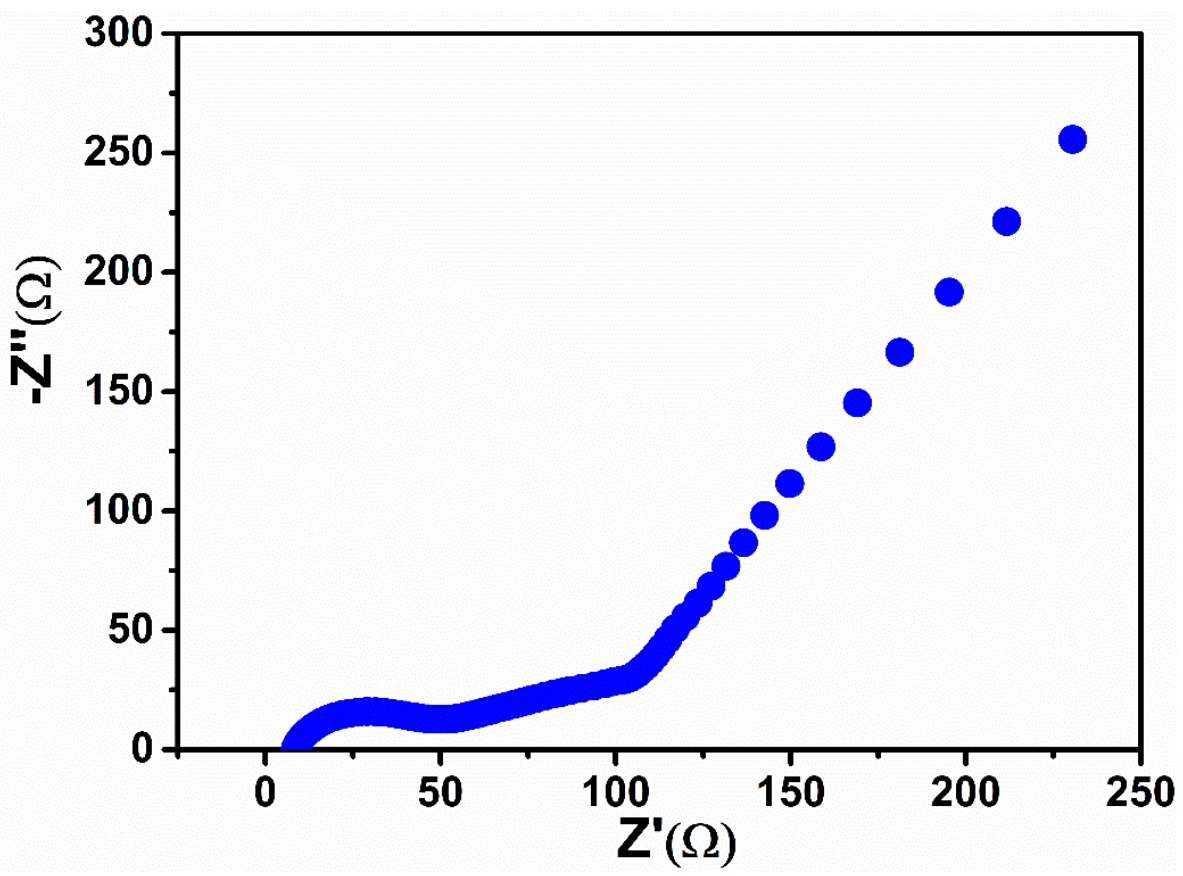

Figure S14. Nyquist plot after cycling. 


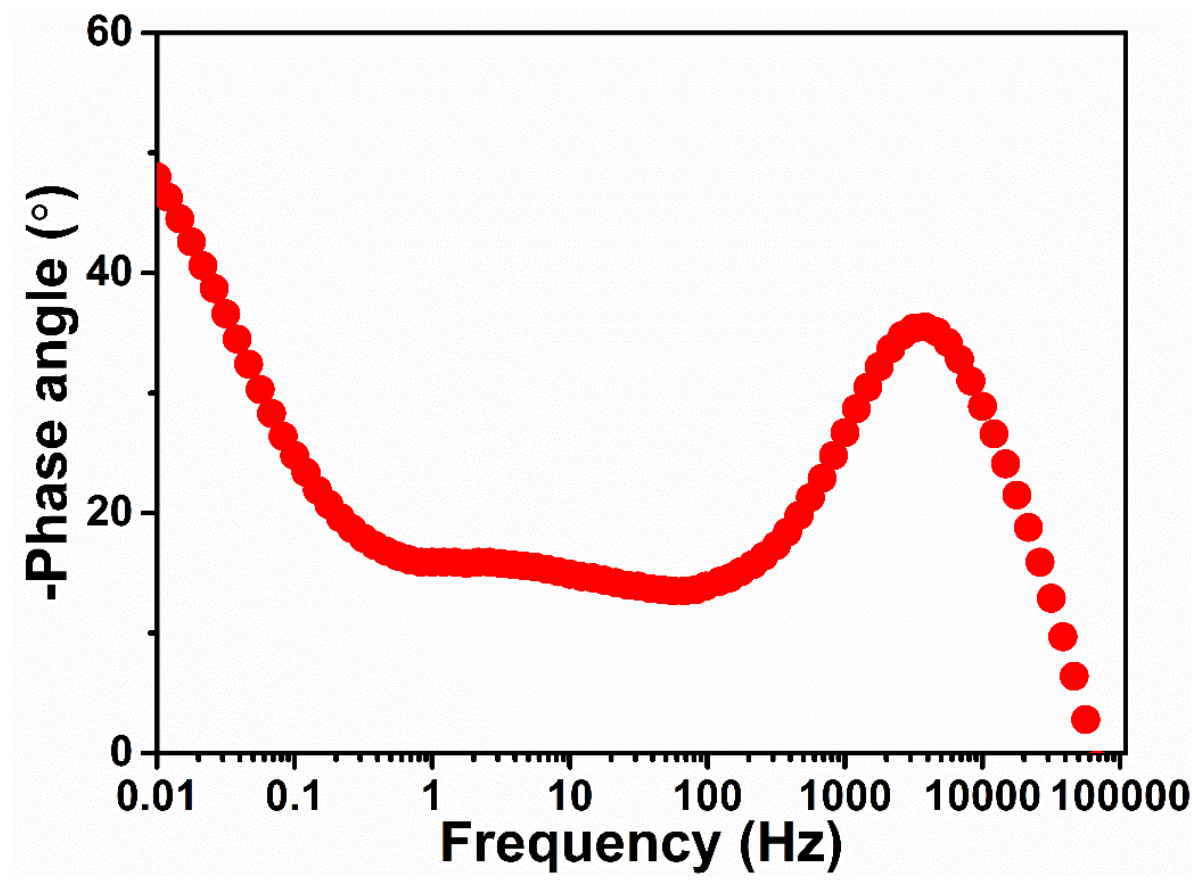

Figure S15. Phase angle with respect to applied frequency after cycling.

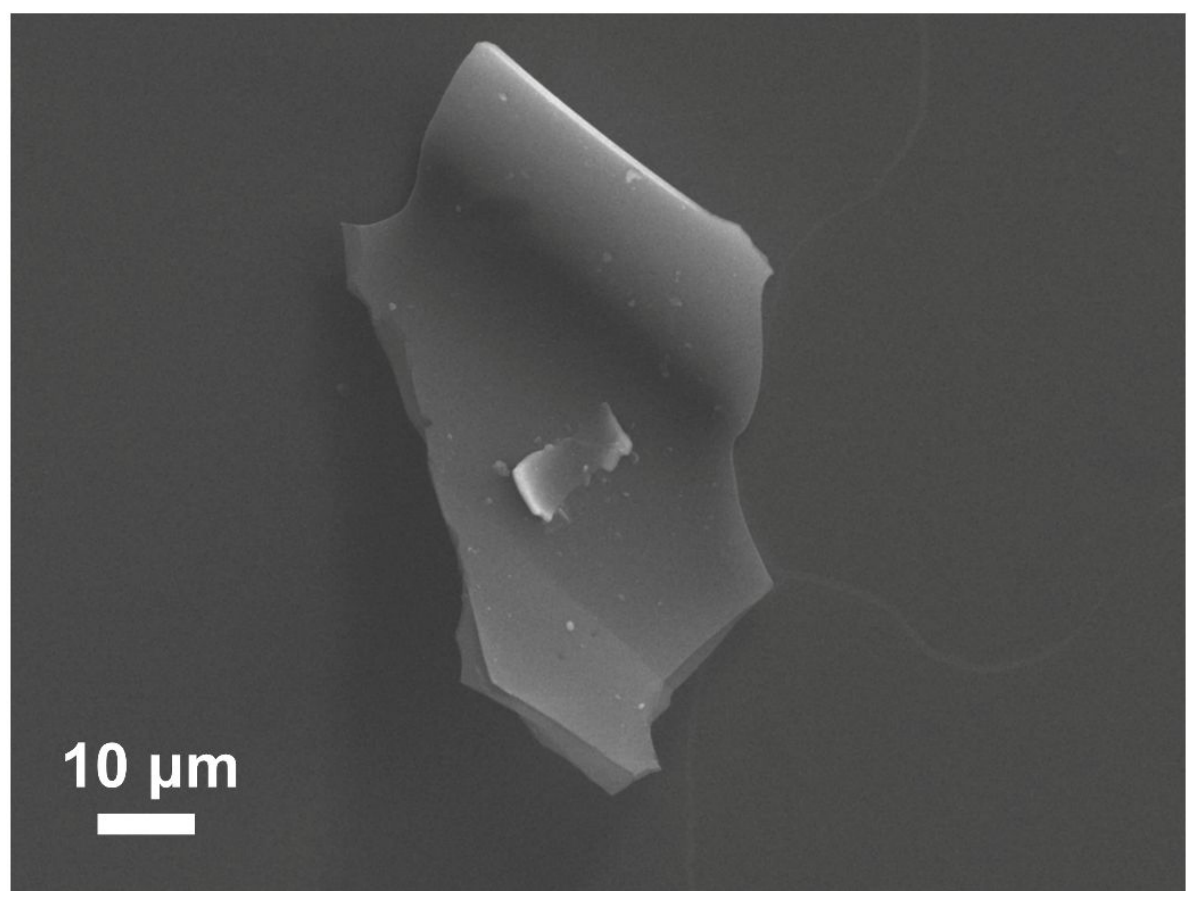

Figure S16. The SEM image of silicene after cycling. 


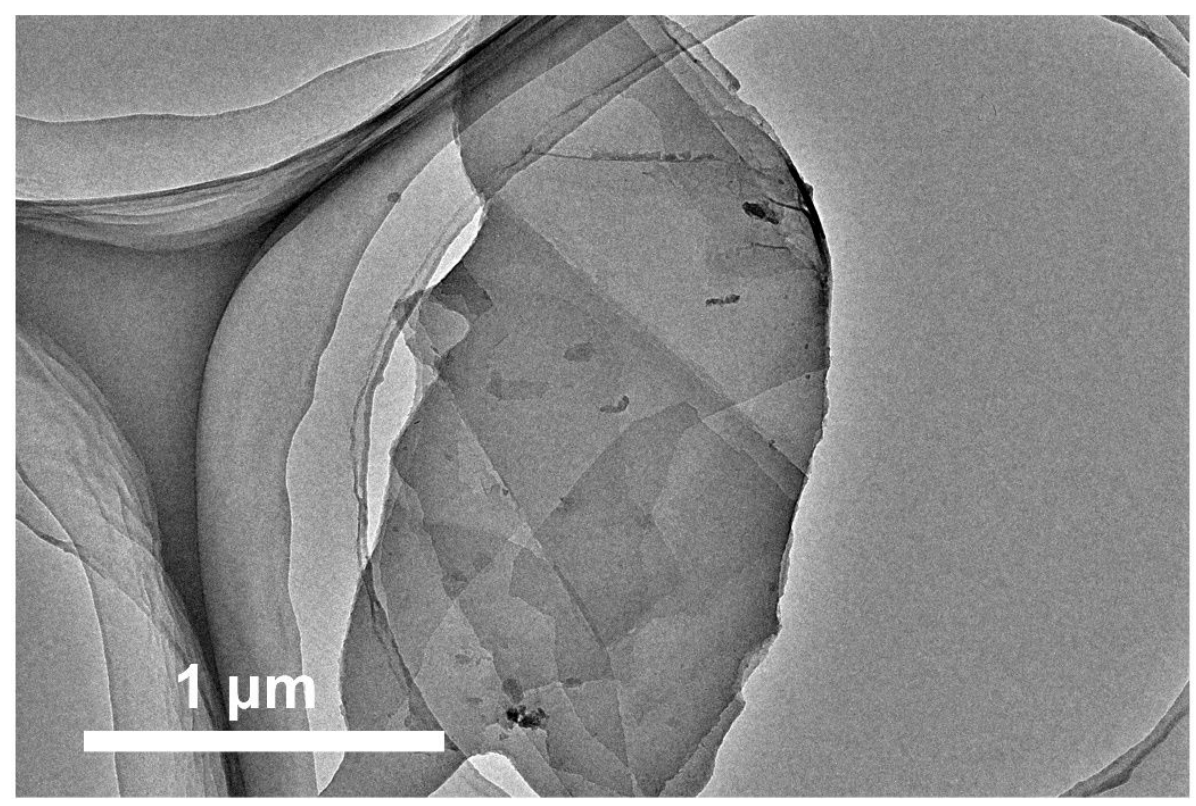

Figure S17. The TEM image of silicene after cycling.

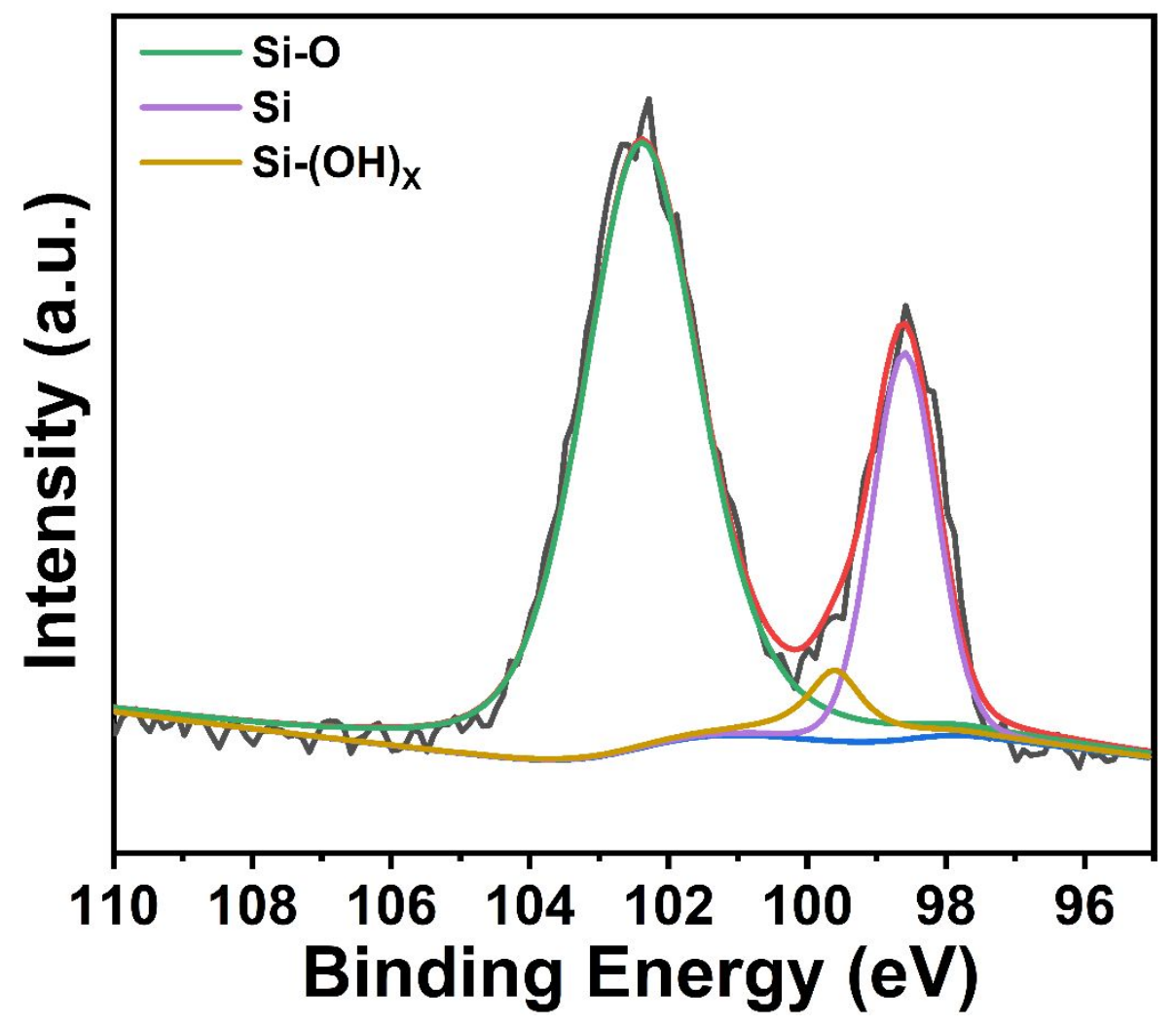

Figure S18. XPS spectrum of silicene in Si 2p region after cycling. 


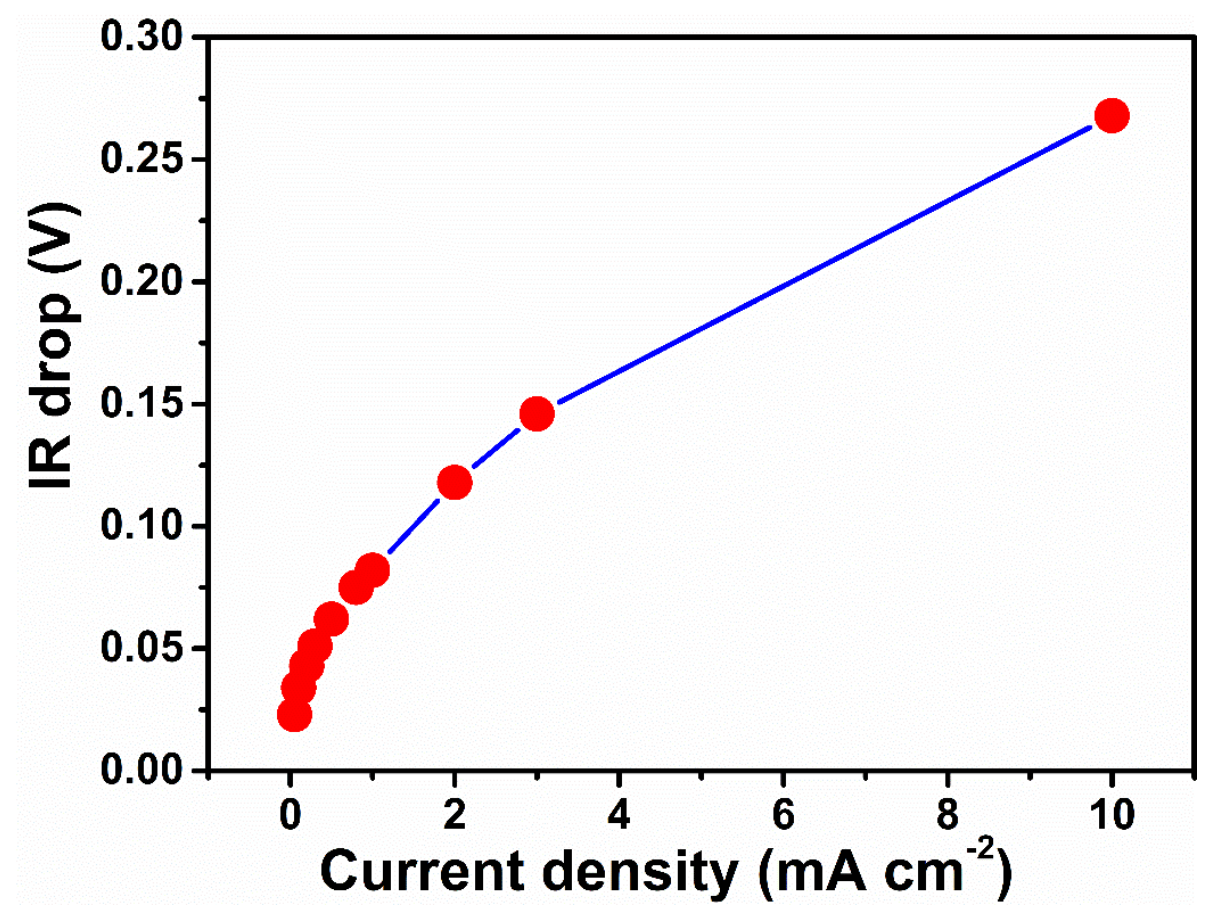

Figure S19. The dependence of IR drop on current density.

Table S1. Comparison of the performance of silicene ZHC and the reported silicon-based ZHC.

\begin{tabular}{|c|c|c|c|c|c|}
\hline Electrode material & Electrolyte & $\begin{array}{c}\text { Potential } \\
\text { window (V) }\end{array}$ & $\begin{array}{c}\text { Specific } \\
\text { capacitance } \\
\left(\mu \mathrm{F} \mathrm{cm}^{-2}\right)\end{array}$ & $\begin{array}{l}\text { Energy } \\
\text { density } \\
\left(\mu \mathrm{J} \mathrm{cm}^{-2}\right)\end{array}$ & 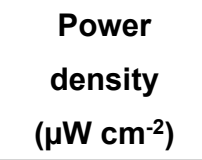 \\
\hline Silicon Nanowire ${ }^{1}$ & PYR $_{13}$ TFSI & 0 to 4 & 30 & 190 & 1000 to 2000 \\
\hline Silicon Nanotrees ${ }^{2}$ & EMI-TFSI & -2.5 to 1.5 & 3800 & 2800 & 2000 \\
\hline $\begin{array}{l}\text { Diamond coated Si } \\
\qquad \mathrm{NWs}^{3}\end{array}$ & PMPyrrTFSI & -2.5 to 1.5 & 105 & 84 & 940 \\
\hline O-Si NWs ${ }^{4}$ & EMI-TFSI & 0 to 4 & 31 & 212 & 472 \\
\hline $\begin{array}{c}\text { PEDOT coated Silicon } \\
\text { nanowire }^{5}\end{array}$ & $\mathrm{PYR}_{13} \mathrm{TFSI}$ & 0 to 1.5 & $8000-9000$ & 9000 & 800 \\
\hline SiC NWs on SiC film ${ }^{6}$ & $\mathrm{KCl}$ & -0.2 to 0.6 & 240 & 68 & 4 \\
\hline SiC coated Si NWs ${ }^{7}$ & $\mathrm{KCl}$ & -0.2 to 0.6 & 1700 & 850 & 100 \\
\hline $\mathrm{SiC} \mathrm{NW}^{8}$ & $\mathrm{KCl}$ & 0.0 to 0.6 & 23000 & 2300 & 1100 \\
\hline Porous $\mathrm{Si}^{9}$ & $\mathrm{PEO} \mathrm{EMIBF}_{4}$ & 0 to 0.65 & 3500 & 612 & 22 \\
\hline $\mathrm{p}-\mathrm{SiNW} \mathbf{s}^{10}$ & $\mathrm{NEt}_{4} \mathrm{BF}_{4}$ & -1.3 to -0.5 & 46 & 3 & 1600 \\
\hline Siloxene ${ }^{11}$ & $\mathrm{TEABF}_{4}$ & 0 to 3.0 & 2180 & 3220 & 4030 \\
\hline Siloxene $^{12}$ & $\mathrm{TEABF}_{4}$ & 0 to 1.8 & 28980 & 12030 & 5620 \\
\hline $\begin{array}{c}\text { Silicene } \\
\text { (This work) }\end{array}$ & $\begin{array}{l}21 \mathrm{M} \mathrm{LiTFSI} \text { and } \\
1 \mathrm{M} \mathrm{Zn}\left(\mathrm{CF}_{3} \mathrm{SO}_{3}\right)_{2}\end{array}$ & 0 to 1.8 & 14220 & 12600 & 9003 \\
\hline
\end{tabular}




\section{References}

1. Aradilla, D.; Gentile, P.; Bidan, G.; Ruiz, V.; Gómez-Romero, P.; Schubert, T.J.S.; Sahin, H.; Frackowiak, E.; Sadki, S. High Performance of Symmetric Micro-Supercapacitors Based on Silicon Nanowires Using N-Methyl-N-Propylpyrrolidinium Bis(trifluoromethylsulfonyl)imide as Electrolyte. Nano Energy 2014, 9, 273-281.

2. Gaboriau, D.; Aradilla, D.; Brachet, M.; Le Bideau, J.; Brousse, T.; Bidan, G.; Gentile, P.; Sadki, S. Silicon Nanowires and Nanotrees: Elaboration and Optimization of New 3D Architectures for High Performance On-Chip Supercapacitors. RSC Adv. 2016, 6, 81017-81027.

3. Gao, F.; Lewes-Malandrakis, G.; Wolfer, M. T.; Müller-Sebert, W.; Gentile, P.; Aradilla, D.; Schubert, T.; Nebel, C. E. Diam. Diamond-Coated Silicon Wires for Supercapacitor Applications in Ionic Liquids. Diamond Relat. Mater. 2015, 51, 1-6.

4. Berton, N.; Brachet, M.; Thissandier, F.; Le Bideau, J.; Gentile, P.; Bidan, G.; Brousse, T.; Sadki, S. Wide-Voltage-Window Silicon Nanowire Electrodes for Micro-Supercapacitors via Electrochemical Surface Oxidation in Ionic Liquid Electrolyte. Electrochem. commun. 2014, 41, 31-34.

5. Aradilla, D.; Bidan, G.; Gentile, P.; Weathers, P.; Thissandier, F.; Ruiz, V.; GomezRomero, P.; Schubert, T. J. S.; Sahin, H.; Sadki, S. Novel Hybrid Micro-Supercapacitor Based on Conducting Polymer Coated Silicon Nanowires for Electrochemical Energy Storage. RSC Adv. 2014, 4, 26462-26467.

6. Alper, J. P.; Kim, M. S.; Vincent, M.; Hsia, B.; Radmilovic, V.; Carraro, C.; Maboudian, R. Silicon Carbide Nanowires as Highly Robust Electrodes for Micro-Supercapacitors. J. Power Sources 2013, 230, 298-302.

7. Alper, J. P.; Vincent, M.; Carraro, C.; Maboudian, R. Silicon Carbide Coated Silicon Nanowires as Robust Electrode Material for Aqueous Micro-Supercapacitor. Appl. Phys. Lett. 2012, 100, 163901.

8. Gu, L.; Wang, Y.; Fang, Y.; Lu, R.; Sha, J. Performance Characteristics of Supercapacitor Electrodes Made of Silicon Carbide Nanowires Grown on Carbon Fabric. J. Power Sources 2013, 243, 648-653.

9. Cohn, A. P.; Erwin, W. R.; Share, K.; Oakes, L.; Westover, A. S.; Carter, R. E.; Bardhan, R.; Pint, C. L. All Silicon Electrode Photocapacitor for Integrated Energy Storage and Conversion. Nano Lett. $2015,15,2727-2731$.

10. Thissandier, F.; Le Comte, A.; Crosnier, O.; Gentile, P.; Bidan, G.; Hadji, E.; Brousse, T.; Sadki, S.; Highly Doped Silicon Nanowires Based Electrodes for Micro-Electrochemical Capacitor Applications. Electrochem. Commun. 2012, 25, 109-111.

11. Krishnamoorthy, K.; Pazhamalai, P.; Kim, S.-J. Two-Dimensional Siloxene Nanosheets: Novel HighPerformance Supercapacitor Electrode Materials. Energy Environ. Sci. 2018, 11, 1595-1602.

12. Krishnamoorthy, K.; Pazhamalai, P.; Mariappan, V. K.; Nardekar, S. S.; Sahoo, S.; Kim, S.-J. Probing the Energy Conversion Process in Piezoelectric-Driven Electrochemical Self-Charging Supercapacitor Power Cell Using Piezoelectrochemical Spectroscopy. Nat. Commun. 2020, 11, 2351. 